\title{
LA REPRESENTACIÓN DE LOS ACTORES SOCIALES DEL REFERÉNDUM EN LA PRENSA ESCRITA: LA NACIÓN Y DIARIO EXTRA ${ }^{1}$
}

\section{THE REPRESENTATION OF SOCIAL ACTORS OF THE REFERENDUM IN THE NEWSPAPER: LA NACIÓNAND DIARIO EXTRA}

\author{
Sindy Mora* \\ Anthony García**
}

RESUMEN

El artículo analiza cómo fueron representados los actores colectivos que participaron en acciones colectivas a favor $y$ en contra del Tratado de Libre Comercio entre Centroamérica, República Dominicana y Estados Unidos (TLC), por la prensa escrita, en particular La Nación y Diario Extra. En el artículo se aprecia cómo en momentos de mayor organización del movimiento del No, justo después de la convocatoria al referéndum por parte del Tribunal Supremo de Elecciones (TSE), tanto Diario Extra como La Nación reportaron un menor número de acciones colectivas relacionadas con el TLC.

PALABRAS CLAVE: COSTA RICA * ACCIONES COLECTIVAS * MEDIOS DE COMUNICACIÓN * REFERÉNDUM * TRATADO DE LIBRE COMERCIO ENTRE CENTROAMÉRICA Y ESTADOS UNIDOS (TLC)

\section{ABSTRACT}

The article analyses how La Nación and Diario Extra characterized political actors who participated in collective actions against and in favor of Central America Free Trade Agreement (CAFTA). The article shows how in the moment in which the NO movement had an important growth, when the Tribunal Supremo de Elecciones (TSE) officially announced the referendum, Diario Extra and La Nación published few collective actions related to CAFTA.

\section{KEYWORDS: COSTA RICA * COLLECTIVE ACTION * MASS MEDIA * REFERENDUM * CENTRAL AMERICA FREE TRADE AGREEMENT (CAFTA)}

$1 \quad$ Este trabajo fue presentado en las V Jornadas de Investigación del Instituto de Investigaciones Sociales de la Universidad de Costa Rica, realizadas durante el mes de setiembre de 2008, y en el panel "Medios de comunicación y estrategias comunicativas de los actores durante el proceso de referéndum en Costa Rica", organizado por el Posgrado Centroamericano de Ciencias Políticas, en octubre de 2008. Agradecemos los comentarios del M.Sc.
Mauricio Herrera, del Dr. Carlos Sandoval y del Dr. Gerardo Hernández a este trabajo.

* Instituto de Investigaciones Sociales de la Universidad de Costa Rica. sindymora@gmail.com

** Instituto de Investigaciones Sociales de la Universidad de Costa Rica. agarcia16@gmail.com 


\section{INTRODUCCIÓN}

En el presente artículo se analizan las formas en las que la prensa escrita costarricense representó a los diversos actores colectivos, que protagonizaron acciones de protesta en la coyuntura del referéndum por el Tratado de Libre Comercio entre Centroamérica, República Dominicana y Estados Unidos (TLC). ¿Cómo fueron representados los distintos actores en oposición o apoyo al Tratado, por los medios de mayor circulación y cobertura nacional?, ¿qué actores fueron visibilizados o invisibilizados por dichos medios?, y ¿cuáles fueron las acciones atribuidas por la prensa a dichos actores?, son las preguntas a las que responde este trabajo.

Por acciones colectivas se entiende las acciones realizadas por dos o más personas 0 grupos, organizados formal o informalmente, que se dirigen a una entidad determinada y que se valen de los recursos de la acción conjunta, a fin de ver satisfecha una demanda. El presente trabajo problematiza la construcción de los actores colectivos hecha desde la prensa escrita, tratando de comprender las lógicas discursivas de construcción de los actores de las protestas. La reconstrucción de estos actores se hace a partir de La Nación (LN) y Diario Extra (DE) ${ }^{2}$.

Es importante señalar que el ámbito de estudio que nos ocupa en este trabajo ha sido analizado por otros autores, en cuyos estudios se ha brindado el análisis de uno de los ciclos de protesta más significativo del país en los últimos 10 años: el Combo ICE (Fonseca, 2003; Martín,

Esta reflexión se inscribe en la actividad de investigación "Seguimiento de las acciones colectivas y grupos organizados en Costa Rica", inscrita en el Instituto de Investigaciones Sociales de la Universidad de Costa Rica, desde el año 2003, en la cual se mantiene un registro permanente de acciones de protesta, a partir de La Nación, Diario Extra y Semanario Universidad. Esta actividad surgió por iniciativa de un grupo de investigadoras del Instituto de Investigaciones Sociales y del Programa Estado de la Nación, y mantiene un registro permanente de acciones clasificadas a partir de un Manual de Codificación de Acciones Colectivas (Mora, 2008). A partir del 2008, la actualización de la Base de Datos se realiza a partir del vínculo establecido con el Observatorio Social de América Latina (OSAL), del Consejo Latinoamericano de Ciencias Sociales (CLACSO).
2004). Un estudio fotográfico de los conflictos sociopolíticos es el que ha realizado Cuvardic (2004) para el caso de la huelga de educadores del 2003. A pesar de estos esfuerzos, los estudios sobre la construcción simbólica de los actores colectivos escasean, más cuando estos actores se encuentran involucrados en procesos de protesta.

Uno de los puntos de partida de este estudio es que muchos de los conflictos que atraviesan a la sociedad costarricense son invisibilizados por la prensa escrita, mientras que otros, como los conflictos laborales o los protagonizados por los trabajadores, a pesar de no encontrarse directamente relacionados con lo laboral, son mayormente visibilizados (Mora, 2008). Siguiendo dicha argumentación, esta tendencia se habría mantenido en el caso de la coyuntura del referéndum por el TLC, considerando el desequilibrio mediático con el que los medios de comunicación comerciales dieron cuenta de la participación de los diversos actores colectivos (Chacón, 2008), y el tipo de actor revelado en las fuentes periodísticas, como se muestra seguidamente.

En este trabajo se realiza un análisis cuantitativo, en el que se exponen las principales tendencias en los abordajes $y$ coberturas de acciones a favor $y$ en contra del TLC, presentados en La Nación (LN) y Diario Extra (DE). Posteriormente, se realiza un análisis de los titulares y de las notas periodísticas en las que ambos medios reportaron la misma acción colectiva, a fin de dar cuenta de las principales diferencias y similitudes en los abordajes periodísticos, los que definen los modelos interpretativos desde los cuales se entiende la participación política (Vasilashis, 1997: 28).

\section{ANÁLISIS CUANTITATIVO DE ABORDAJES Y COBERTURAS DE ACCIONES COLECTIVAS}

Para el año 2007 se registraron un total de 333 acciones colectivas y 365 noticias sobre acciones colectivas, esta diferenciación se explica ya que una acción colectiva puede ser registrada por los diferentes medios utilizados en la reconstrucción de la protesta social. Las noticias seleccionadas para el presente trabajo corresponden a 297 noticias sobre acciones colectivas. La selección de la muestra se hizo según la demanda expresada por los actores, las cuales corresponden a las siguientes categorías: "aprobar el 
TLC", "rechazar el TLC" y "otras demandas". Se excluyeron las noticias que versan sobre el TLC en las cuales su demanda no corresponde a la aprobación o rechazo del mismo.

Como muestra el gráfico 1, los medios seleccionados reportaron más noticias sobre acciones colectivas no relacionadas con el Tratado de Libre Comercio (TLC), es decir, brindaron una mayor cobertura a "otras deman- das", referidas principalmente a la defensa del trabajo y las condiciones laborales, así como a la garantía y el acceso a la educación (García, 2008: 13-14). No obstante, cuando se reportó sobre el TLC, se brindaron más noticias en contra que a favor del Tratado, lo que se encuentra relacionado con las secciones periodísticas utilizadas para la reconstrucción de las acciones colectivas ${ }^{3}$.

\section{GRÁFICO 1}

\section{COSTA RICA: NOTICIAS SOBRE ACCIONES COLECTIVAS SEGÚN RECHAZO O APROBACIÓN} DEL TLC Y OTRAS DEMANDAS. 2007

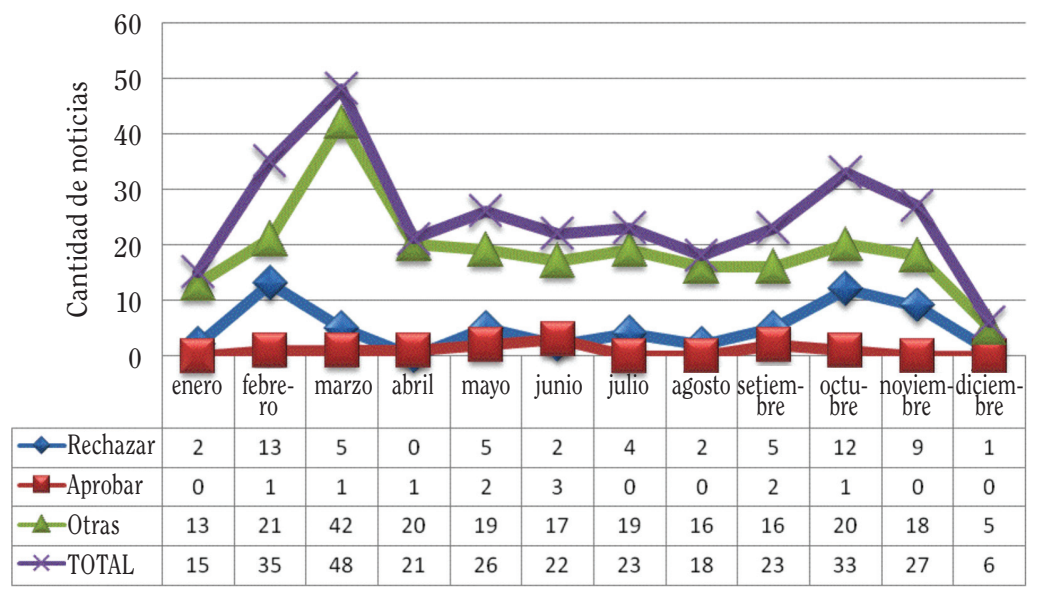

Fuente: Elaboración propia a partir de Diario Extra y La Nación.

Como se observa en la gráfica, en el periodo que comprende los meses de abril a setiembre de 2007, los medios reportaron 19 noticias sobre acciones colectivas referentes al TLC, de un total de 297 noticias analizadas. Esta reducida cobertura contrastó con el alto periodo de movilización social que se desarrolló en estos meses, ya que a partir del 12 de abril de 2007 —cuando el Tribunal Supremo de Elecciones (TSE) acogió la iniciativa del Poder Ejecutivo de llevar a acabo el referéndum para dirimir el conflicto por el TLC_, y el 7 de octubre de 2007 — día de celebración del referéndum-, muchos fueron los actores que se organizaron para posicionarse en torno al Tratado (Raventós, 2008), e incluso, durante este mismo periodo aumentó la intención de voto por el NO, como lo muestran los resultados provenientes de las encuestas realizadas por PROCESOS (Rodríguez, 2008: 5).
Como se observa en el gráfico 2 , durante el año 2007, mensualmente más del 60\% de las noticias reportadas por ambos medios correspondió a la categoría "otras demandas", esto queda aún mas claro en el periodo que comprende a los meses de mayo-agosto 4 en el cual las noticias de esta categoría aumentan del 73,1\% a 88,9\%. Los

3

En particular se consultaron las secciones El país y Vistazo Cantonal en LN y la sección de Nacionales en DE. No fueron tomadas en cuenta la publicidad o campos pagados que permitirían ver la participación de otros actores, con otra disponibilidad de recursos, en cuanto a su posicionamiento en torno al TLC.

4 Después de que el TSE acogió la iniciativa del Poder Ejecutivo de llevar a acabo el referéndum y antes de la celebración del mismo. 
meses en los que se reportó un mayor número de noticias en torno al TLC fueron los meses en los que se desarrollaron las marchas más concurridas (febrero, mayo, setiembre).

\section{GRÁFICO 2}

\section{COSTA RICA: NOTICIAS SOBRE ACCIONES COLECTIVAS DE RECHAZO O APROBACIÓN DEL TLC Y OTRAS DEMANDAS, SEGÚN FUENTE PERIODÍSTICA. 2007}

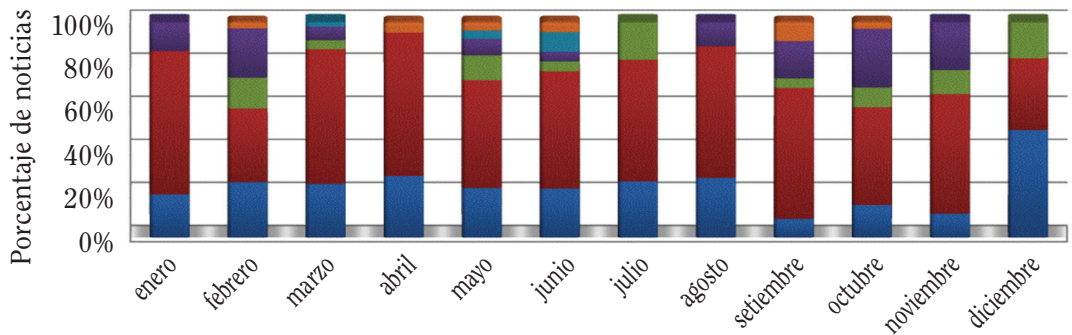

\begin{tabular}{|c|c|c|c|c|c|c|c|c|c|c|c|c|}
\hline & enero & $\begin{array}{c}\text { febre- } \\
\text { ro }\end{array}$ & marzo & abril & mayo & junio & julio & agosto & $\begin{array}{l}\text { setiem- } \\
\text { bre }\end{array}$ & $\begin{array}{l}\text { octu- } \\
\text { bre }\end{array}$ & $\begin{array}{c}\text { novien } \\
\text { bre }\end{array}$ & $\begin{array}{c}\text { diciem- } \\
\text { bre }\end{array}$ \\
\hline -Diario Extra (a favor) & 0 & 1 & 0 & 1 & 1 & 1 & 0 & 0 & 2 & 1 & 0 & 0 \\
\hline - La Nación (a favor) & 0 & 0 & 1 & 0 & 1 & 2 & 0 & 0 & 0 & 0 & 0 & 0 \\
\hline -Diario Extra (oposición) & 2 & 8 & 3 & 0 & 2 & 1 & 0 & 2 & 4 & 9 & 6 & 0 \\
\hline -La Nación (oposición) & 0 & 5 & 2 & 0 & 3 & 1 & 4 & 0 & 1 & 3 & 3 & 1 \\
\hline Diario Extra (otras) & 10 & 12 & 30 & 14 & 13 & 12 & 13 & 11 & 14 & 15 & 15 & 2 \\
\hline - La Nación (otras) & 3 & 9 & 12 & 6 & 6 & 5 & 6 & 5 & 2 & 5 & 3 & 3 \\
\hline
\end{tabular}

Fuente: Elaboración propia a partir de Diario Extra y La Nación

El gráfico 3 muestra estas mismas tendencias para el caso del Diario Extra (DE). Como se puede observar, este medio dedicó un mayor número de sus espacios al reporte de noticias con demandas no rela- cionadas con el TLC. Cuando se atendieron las demandas relacionadas con el Tratado, se reportaron más acciones en contra que a favor, principalmente en febrero y noviembre.

\section{GRÁFICO 3}

COSTA RICA: NOTICIAS SOBRE ACCIONES COLECTIVAS DE RECHAZO O APROBACIÓN DEL TLC Y OTRAS DEMANDAS, SEGÚN DIARIO EXTRA. 2007

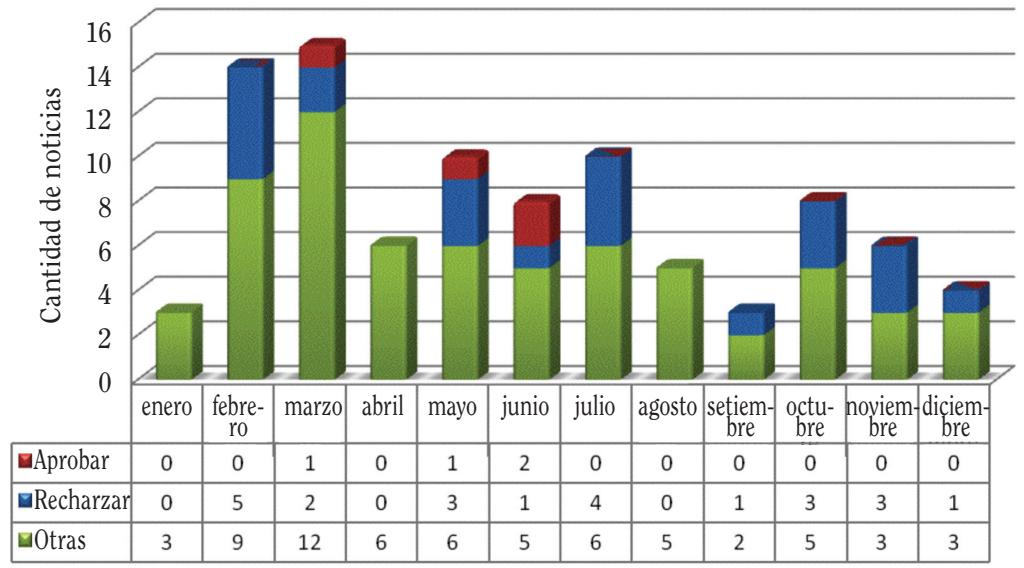

Fuente: Elaboración propia a partir de Diario Extra y La Nación 
En el caso de La Nación (LN), el gráfico 4 muestra las mismas tendencias reseñadas para DE: se reportaron más acciones de protesta no relacionadas con el TLC, correspondiendo mensualmente a más del 50\%, y en los meses de enero, abril y agosto a un $100 \%$. Los únicos meses en los que se reportaron noticias sobre acciones colectivas a favor del TLC fueron marzo, mayo y junio, este último mes con el mayor número. Para los meses de setiembre, octubre $y$ noviembre - antes $y$ después del referéndum - se reportaron un mayor número de noticias en términos relativos. Cuando se trató el TLC, se reportaron más acciones en contra que a favor del mismo.

\section{GRÁFICO 4}

COSTA RICA: NOTICIAS SOBRE ACCIONES COLECTIVAS DE RECHAZO O APROBACIÓN DEL TLC Y OTRAS DEMANDAS, SEGÚN LA NACIÓN. 2007

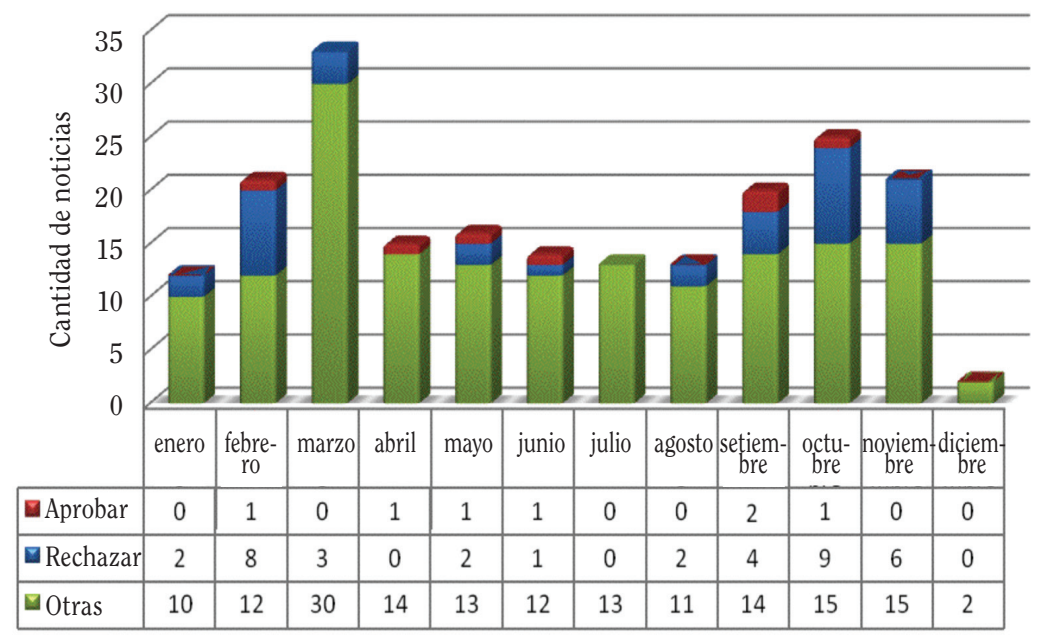

Fuente: Elaboración propia a partir de Diario Extra y La Nación.

El gráfico 5 muestra que Diario Extra (DE) publicó menos noticias sobre acciones colectivas de oposición al Tratado, que La Nación (LN). En el periodo que comprende los meses de abril a setiembre, la cantidad de noticias registradas corresponde a 18 , es decir un $30 \%$ del total de noticias de oposición al TLC durante el año. Este periodo como se mencionó anteriormente es el más álgido y como lo demuestra el estudio de PROCESOS, la evolución de intención del voto por el NO aumentó, lo cual indica que los medios de comunicación no dieron cuenta y/o invisibilizaron mediante el registro de "otras demandas" (esta categoría aumentó para ese periodo de un $73,1 \%$ a un $89,1 \%$ ) la protesta social. Los meses en los que se reportó una mayor cantidad de noticias en oposición al TLC coinciden con las marchas más relevantes que se produjeron en el año (febrero, mayo, octubre). Estos meses representan el 50\% de las noticias de oposición al TLC. 


\section{GRÁFICO 5}

COSTA RICA: NOTICIAS SOBRE ACCIONES COLECTIVAS DE OPOSICIÓN AL TLC, SEGÚN FUENTE PERIODÍSTICA. 2007

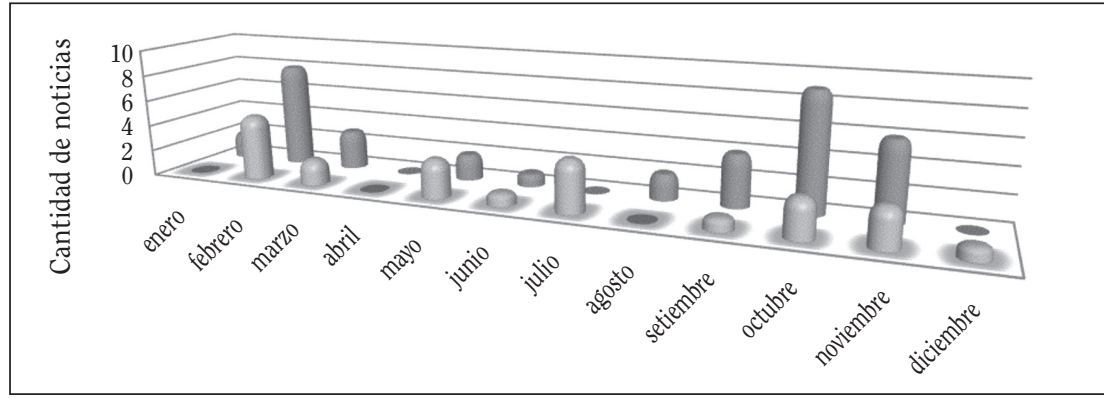

\begin{tabular}{|c|c|c|c|c|c|c|c|c|c|c|c|c|}
\hline & enero & $\begin{array}{c}\text { febre- } \\
\text { ro }\end{array}$ & marzo & abril & mayo & junio & julio & agosto & $\begin{array}{l}\text { setiem- } \\
\text { bre }\end{array}$ & $\begin{array}{l}\text { octu- } \\
\text { bre }\end{array}$ & $\begin{array}{l}\text { hoviem } \\
\text { bre }\end{array}$ & $\begin{array}{c}\text { diciem- } \\
\text { bre }\end{array}$ \\
\hline La Nación (oposición) & 0 & 5 & 2 & 0 & 3 & 1 & 4 & 0 & 1 & 3 & 3 & 1 \\
\hline Diario Extra (oposición) & 2 & 8 & 3 & 0 & 2 & 1 & 0 & 2 & 4 & 9 & 6 & 0 \\
\hline
\end{tabular}

Fuente: Elaboración propia a partir de Diario Extra y La Nación.

Así mismo, el gráfico 6 muestra que setiembre fue el mes en que DE publicó más acciones a favor del TLC. Este medio publicó un mayor número de noticias (7), superando a LN en un $75 \%$ en cuanto noticias a favor. Las noticias a favor de la aprobación del Tratado sumaron un total de 11, y en el periodo que se analizó en los gráficos 2 y 5 (mayo-agosto) se registraron un total de 7 noticias en ambos periódicos, es decir $63,64 \%$ del total de noticias a favor del TLC durante el año. Según el trabajo realizado por PROCESOS, la evolución de la intención del voto por el sí para este periodo disminuyó, lo cual contrasta con las noticias registradas por los medios. En los meses de enero, julio, agosto, noviembre $y$ diciembre no se registró ninguna acción a favor de la aprobación del Tratado.

\section{GRÁFICO 6}

COSTA RICA: NOTICIAS SOBRE ACCIONES COLECTIVAS A FAVOR DEL TLC, SEGÚN FUENTE PERIODÍSTICA. 2007

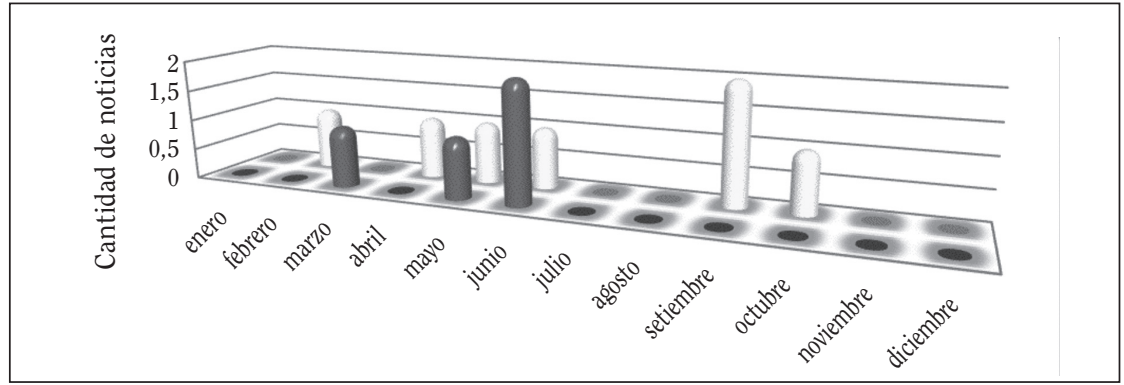

\begin{tabular}{|c|c|c|c|c|c|c|c|c|c|c|c|c|}
\hline & enero & $\begin{array}{c}\text { febre- } \\
\text { ro }\end{array}$ & marzo & abril & mayo & junio & julio & agosto & $\begin{array}{l}\text { setiem- } \\
\text { bre }\end{array}$ & $\begin{array}{l}\text { octu- } \\
\text { bre }\end{array}$ & $\begin{array}{l}\text { noviem } \\
\text { bre }\end{array}$ & $\begin{array}{l}\text { diciem- } \\
\text { bre }\end{array}$ \\
\hline La Nación (a favor) & 0 & 0 & 1 & 0 & 1 & 2 & 0 & 0 & 0 & 0 & 0 & 0 \\
\hline 14 Diario Extra (a favor) & 0 & 1 & 0 & 1 & 1 & 1 & 0 & 0 & 2 & 1 & 0 & 0 \\
\hline
\end{tabular}

Fuente: Elaboración propia a partir de Diario Extra y La Nación. 
El periodo de los meses de mayo a setiembre de los gráficos 5 y 6 queda resumido en el siguiente organigrama:

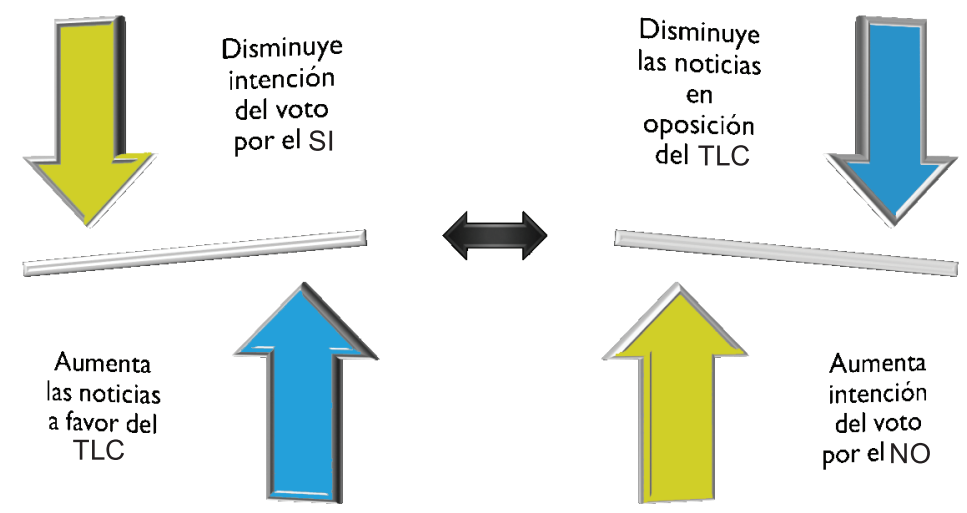

\section{LOS ACTORES EN LA PRENSA ESCRITA ¿CÓMO FUERON REPRESENTADOS?}

Desde la perspectiva de la prensa, ¿cómo fueron representados los actores sociales en la prensa escrita? y ¿cuáles fueron las acciones realizadas por estos actores? Siguiendo las líneas metodológicas propuestas por Vasilachis (1997: 35) para la identificación de actores sociales, en el presente apartado analizamos la construcción periodística de los actores y las acciones atribuidas por la prensa a dichos actores.

Al analizar los titulares de las noticias en las que se reportaron acciones colectivas de protesta relacionadas con el TLC, encontramos diferencias significativas en los medios periodísticos consultados. En el caso de La Nación (LN), los actores identificados en los titulares fueron los que se presentan en el cuadro 1: El NO, sindicatos, opositores; El sí, trabajadores, Frente de Lucha contra el TLC, organizadores, rectores, TSE, Iglesia. Como se puede observar, las acciones colectivas asociadas al "NO", son las de grupos que apuntan a unificarse, pero cuyos intentos son fallidos, dada la conflictividad que les caracteriza. De acuerdo con la lectura de la LN, esta conflictividad termina por imponerse en las prácticas organizativas de este grupo ("Conflictiva reunión del NO" (LN 09/10/07)). En contraposición, "El sí" fue retratado en los titulares como un grupo heterogéneo, que "se une", y que reitera "apoyos" para el trabajo conjunto ("Grupo heterogéneo se une para el sí" (LN 05/06/07)). Las acciones más confrontativas desde la perspectiva de LN fueron las asignadas a los sindicatos, a los opositores y a los trabajadores, como se muestra en el cuadro 1 (p. 96). Son los sindicatos los que advirtieron sobre un eventual choque social, $y$ son quienes anunciaron paros $y$ marchas ("ANEP advierte sobre choque social" (LN 01/03/07)). Por su parte, a los opositores se les adjudicaron acciones como el desconocimiento de legitimidad y el boicot, siendo los trabajadores, los actores identificados con la agresividad ("Opositores al TLC boicotearían referendo si se votan leyes paralelas" (LN 11/07/07), "Grupo anti-TLC desconoce legitimidad de Sala IV y TSE" (LN 06/07/07), "Agresivo paro de labores tuvo mínimo apoyo en el ICE" (LN 27/11/07)).

A diferencia de La Nación (LN), Diario Extra (DE) registró una mayor diversidad de actores, como se puede observar en el cuadro 2 (p. 97). Esta diversidad incluyó a Sindicatos, Trabajadores, Los del No, Opositores al TLC, Sectores sociales, Universitarios, Porteadores, Indígenas, Empresarios, Campesinos, Rectores, TSE y Comités Patrióticos. Para DE, las acciones colectivas relacionadas con la violencia son las que se encuentran en manos de los trabajadores ("Llaman a rebelión en huelga del ICE" (DE 17/11/07)) y los del NO, quienes atacan las casas de los diputados ("A semillazos los del "NO" 


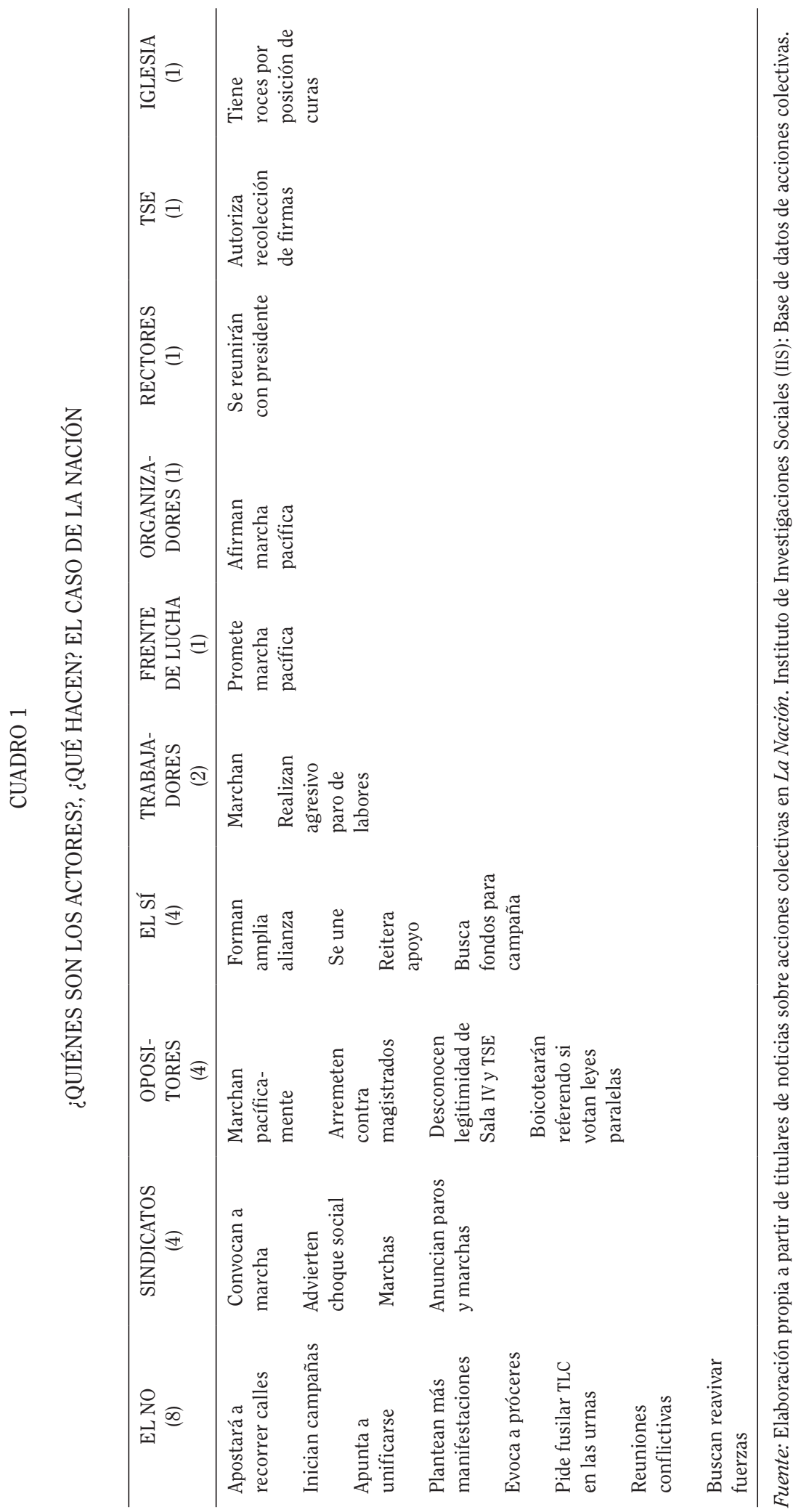




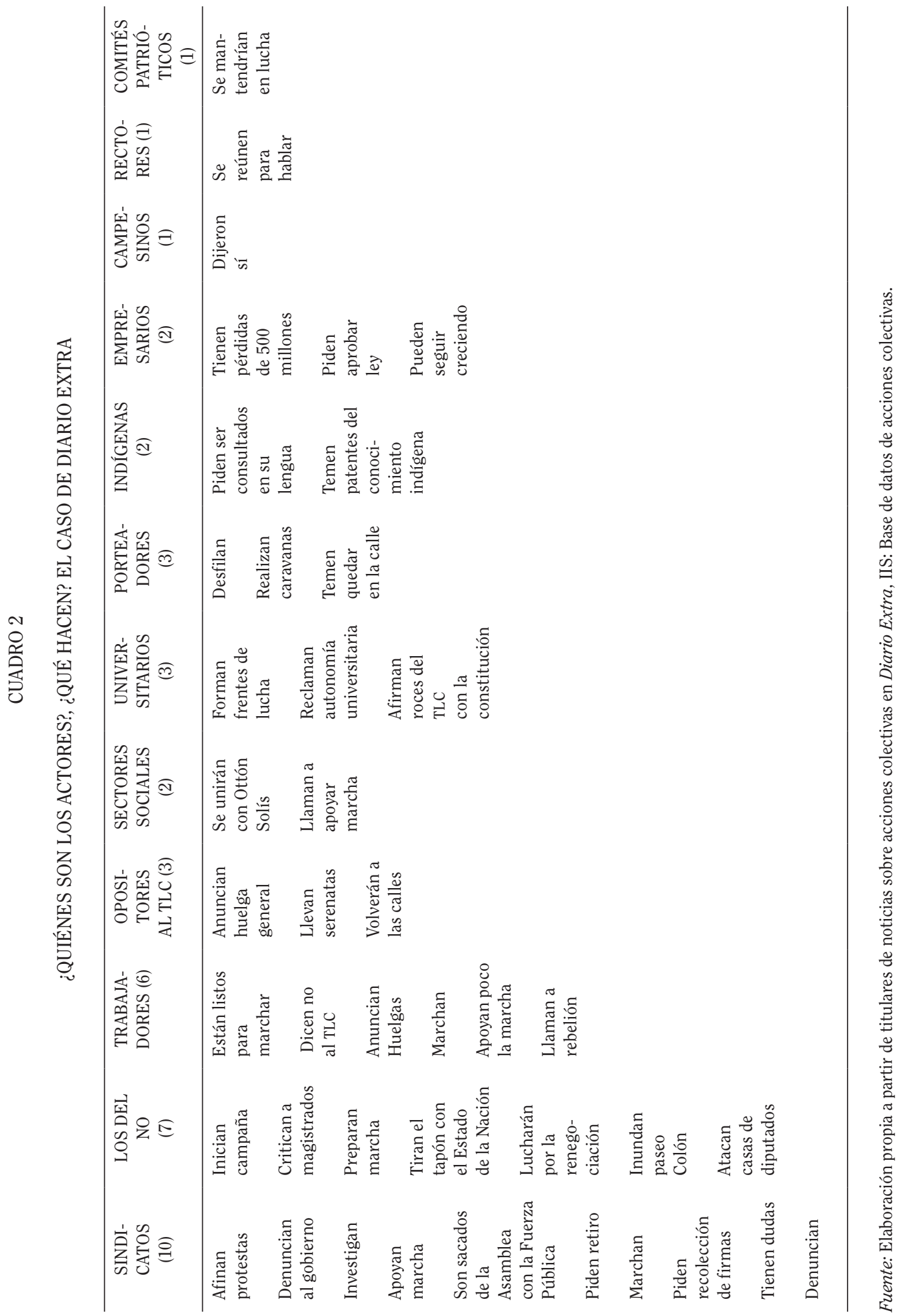


atacan casas de diputados" (DE 9/11/07)). Fue DE el medio que hizo mención a los Comités Patrióticos en un único titular, y después de la realización del referéndum, señalando que estos grupos sociales son los que se mantendrán en lucha ("Comités Patrióticos de todo el país se mantendrán en lucha" (DE 12/10/07)).

La variable de los perdedores entró a jugar en los titulares de Diario Extra (DE). De acuerdo con la nota periodística, los empresarios son los perdedores con la realización de protestas, quienes tienen pérdidas económicas, pero al mismo tiempo tienen las posibilidades de seguir creciendo ante una eventual aprobación del TLC ("Pérdidas pueden ser de hasta \$500 millones” (DE 27/02/07)), (“El TLC es el marco de referencia para seguir creciendo" (DE 23/04/07)).

Ahora bien, ¿cómo fueron reconstruidos los actores en los textos de las noticias? Tanto en LN como en DE se reportaron un total de 6 noticias, en las que se informó sobre las mismas acciones colectivas protesta. Estas acciones fueron (1) la convocatoria a la marcha del 26 de febrero, (2) las marchas del 26 de febrero, (3) del 2 de mayo y (4) del 30 de setiembre, (5) el anuncio de la reunión entre Eugenio Trejos, del Frente Nacional de Apoyo a la Lucha contra el TLC, y el presidente de la República, Óscar Arias, y (6) las reacciones de los actores después del pronunciamiento de la Sala IV, sobre la consulta de constitucionalidad sobre el TLC. Los titulares de estas noticias son las que se presentan en el cuadro 3 (ps. 99, 100 y 101).

De acuerdo con la información sistematizada a partir de notas periodísticas, ¿quiénes fueron los actores que participaron mediante los recursos de la acción colectiva en el proceso del referéndum del 2007? Los abordajes periodísticos construyeron un protagonismo que no necesariamente reflejó la diversidad de actores organizados formal o informalmente en las protestas. En términos generales, se puede decir que la prensa retrató un movimiento de oposición al Tratado, que fue encabezado por los partidos políticos con representación en la Asamblea Legislativa. De esta forma, los diputados y diputadas del PAC, el FA y el PASE, cuyos representantes participaron en marchas y/o declaraciones públicas, fueron representados como los principales organizadores y promotores de estas acciones colectivas. De acuerdo con el discurso de la prensa, quienes también protagonizaron este proceso fueron las organizaciones de trabajadores sindicalizados, principalmente del ICE, $y$ otras organizaciones magisteriales, como el SEC, la ANDE o el APSE. En estas noticias no tuvo mayor relevancia el papel desempeñado por las organizaciones constituidas para organizar políticamente la oposición al TLC, como la Comisión Nacional de Enlace, o el Frente Nacional de Apoyo a la Lucha contra el TLC.

En estas noticias se hicieron evidentes algunas de las tendencias señaladas en el caso de los titulares: la reducida referencia a las mujeres que participaron en política, y cuando se hizo, se mencionó a las diputadas o integrantes del PAC, así como a la exdiputada socialcristiana Gloria Valerín. También se desdibujaron de las notas periodísticas otros grupos, como los Comités Patrióticos o cualquier otra forma de organización comunal o vecinal, las organizaciones de carácter religioso, independientemente de la denominación, así como las organizaciones de mujeres, como las Mujeres de Blanco.

Adicionalmente, en estas noticias predominó el uso de la identificación de determinadas personalidades de la política nacional, entre las que se destacaron los miembros del Partido Acción Ciudadana, disidentes del Partido Liberación Nacional, representantes de la oposición gubernamental en la Asamblea Legislativa y sindicalistas. Estas personalidades son las que se citan a continuación ${ }^{5}$ : Eugenio Trejos del Frente Nacional de Lucha (11), Monseñor Ignacio Trejos, Obispo Emérito de San Isidro de El General (11), José Miguel Corrales, excandidato presidencial del PLN (9), Rodrigo Carazo, expresidente de la República (4), Ottón Solís del PAC (4), José Merino del FA (3), Óscar López del PASE (3), Jorge Arguedas del Fit (3), Édgar Morales de la ANEP (3), Alberto Salom (2), Elizabeth Fonseca (2) y

$5 \quad$ Entre paréntesis se presenta el número de veces en que fueron citados en estas noticias. 


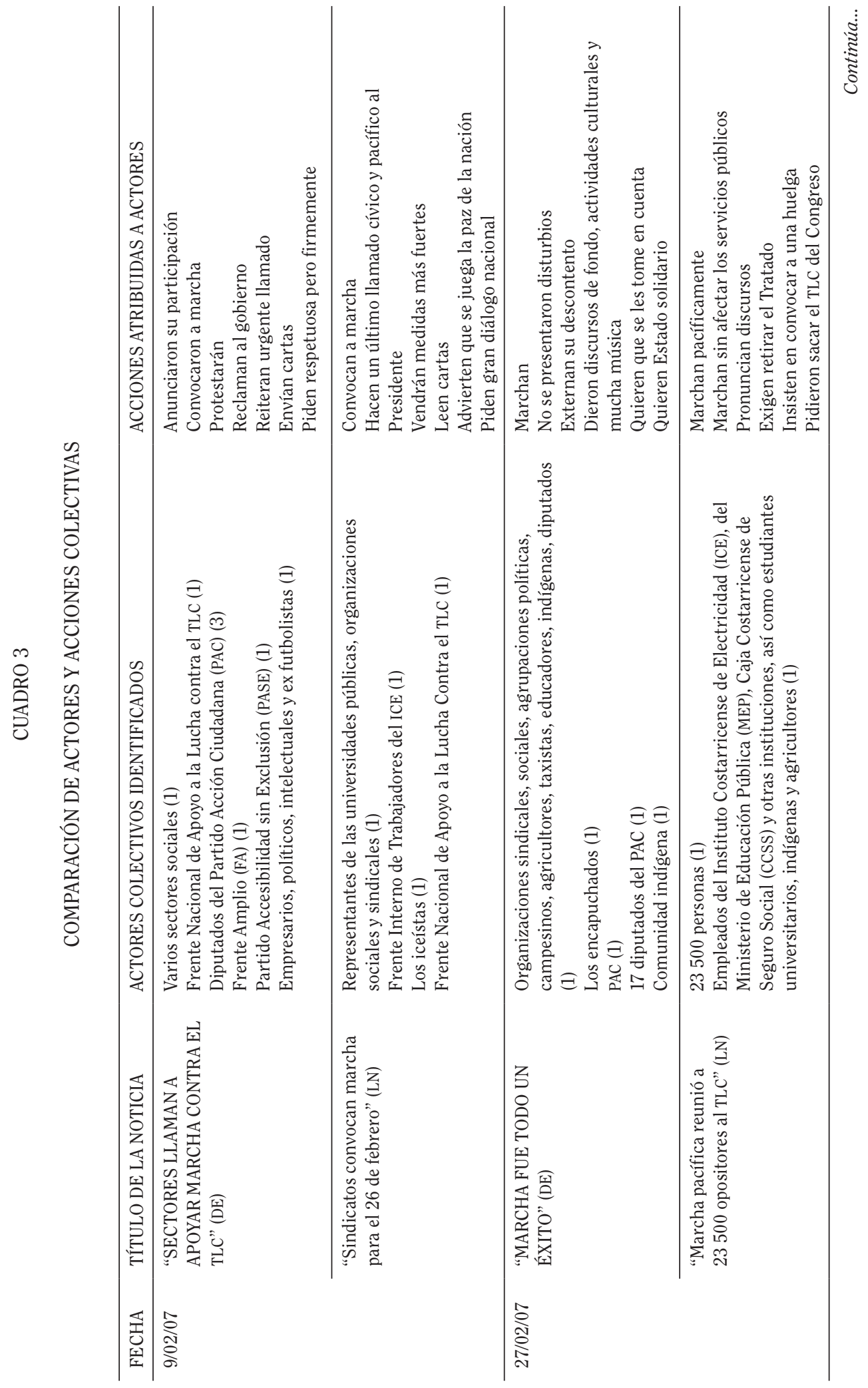




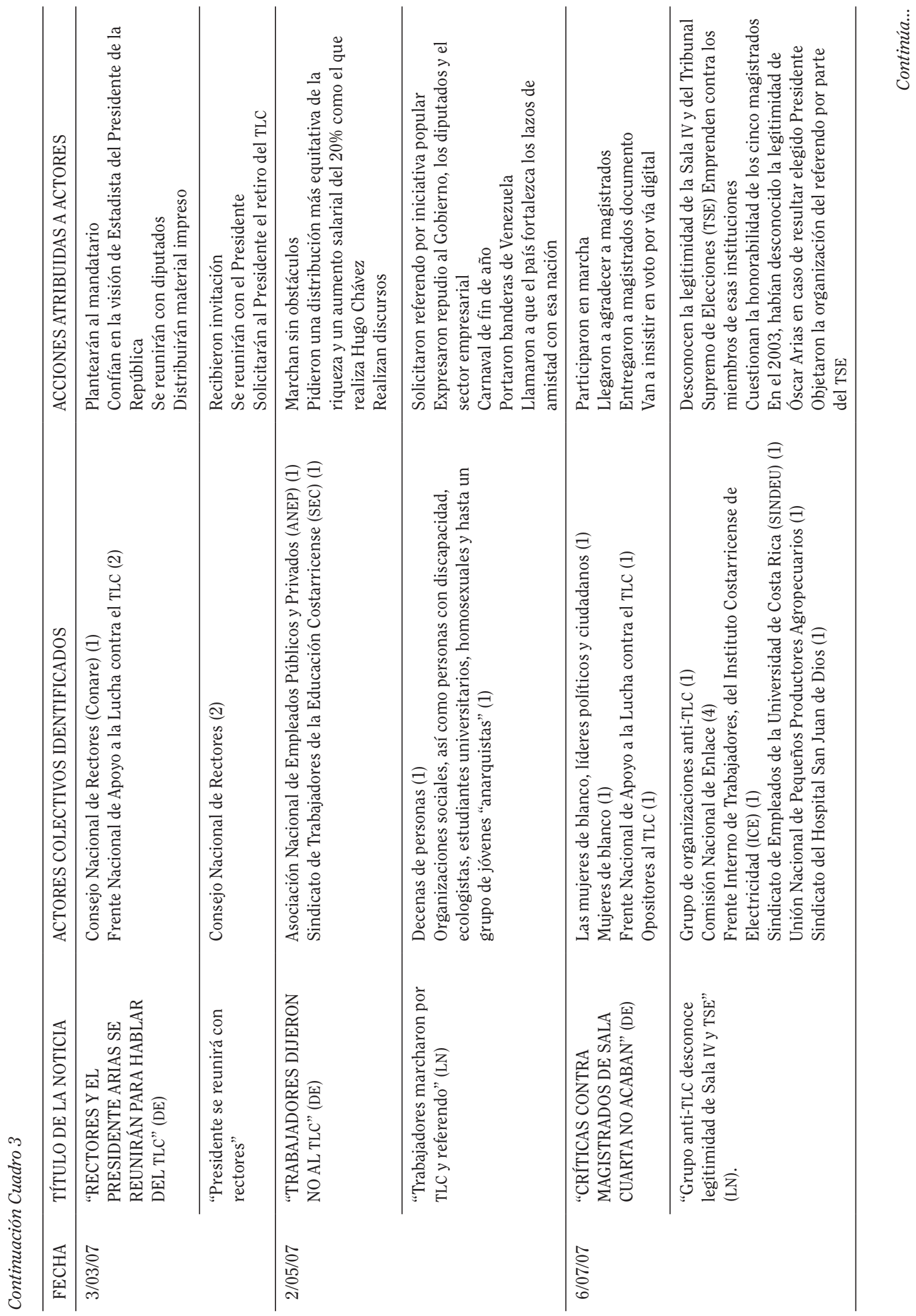




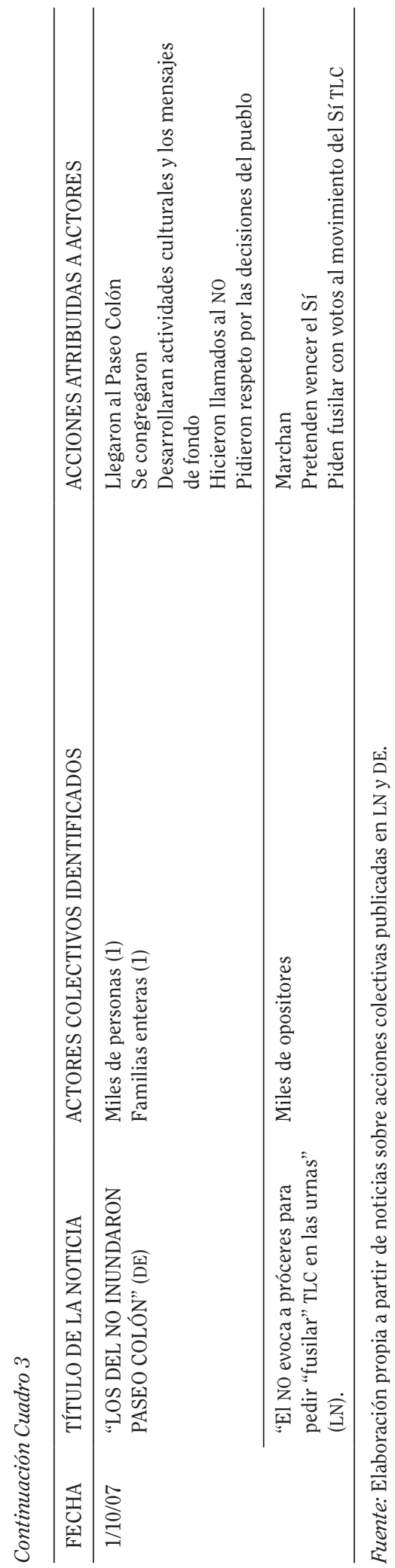


Andrea Morales (2), diputados del PAC, Gilbeth Díaz del SEc (2), Gloria Valerín, exdiputada del PUSC (2), Rolando Araya, excandidato presidencial del PLN (1), Luis Alberto Monge, expresidentes de la República (1), Epsy Campbell del PAC (1), Mariano Figueres, exintegrante del PLN (1), Benjamin Mayorga, exfutbolista (1), Jesús Vásquez de la APSE (1), Luis Salas del INS (1) y Henry Mora, catedrático de la Universidad Nacional (1).

De acuerdo con los medios consultados, ¿qué acciones realizaron estos actores? Ambos medios de comunicación hicieron referencia a las acciones de organización de protesta o a la protesta en sí, como la convocatoria a marchas o huelgas $y$ los eventuales encuentros entre el gobierno y los "representantes" del NO, $y$ las críticas a los actores a instituciones como el TSE $y$ la Sala IV.

Sin embargo, una diferencia significativa entre los abordajes de La Nación (LN) y Diario Extra (DE) fue el énfasis que el primer medio puso en los calificativos pacíficos de las acciones de protesta, o de los manifestantes. Esto se puede observar en el cuadro 3, en donde, de acuerdo con LN, los actores hicieron "un último llamado cívico y pacífico al Presidente", "Advierten que se juega la paz de la nación" y "Piden gran diálogo nacional", ("Sindicatos convocan marcha para el 26 de febrero" (LN 9/02/07)), "Marchan pacíficamente", "Marchan sin afectar los servicios públicos" ("Marcha pacífica reunió a 23.500 opositores al TLC" (LN 27/02/07)).

Esta diferencia resulta más clara con las noticias correspondientes al 6 de julio de 2007, como se observa en el cuadro 3 , en donde los actores representados en DE, que se pronunciaron en contra de la resolución de la Sala IV, "participaron en la marcha", "llegaron a agradecer a magistrados" que encontraron vicios de constitucionalidad en el texto del Tratado, "entregaron a los magistrados un documento" con la posición del Movimiento del NO, y "demandaron el voto por vía digital" ("Críticas contra magistrados de sala cuarta no acaban" (DE 06/07/07)). En contraposición a esta reconstrucción periodística, los actores representados por LN, "desconocieron la legitimidad de la Sala
IV y del TSE", "objetaron la organización del referendo por parte del TSE", "la emprendieron contra los miembros de esas instituciones", "cuestionaron la honorabilidad de los cinco magistrados", y ya en el 2003, "habían desconocido la legitimidad de Óscar Arias en caso de resultar elegido Presidente de la República" ("Grupo anti-TLC desconoce legitimidad de Sala IV y TSE" (LN 06/07/07)).

El énfasis que La Nación (LN) puso en el carácter pacífico de las protestas o en su potencial violencia, $y$ en las críticas a las instituciones públicas, evidencia que existe un nivel en la institucionalidad que no debe ser cuestionado ni criticado, como lo serían las instituciones organizadoras del juego político electoral, o la Sala IV. En las noticias analizadas, este aspecto no fue presentado en DE.

El abordaje del DE muestra diferencias en la construcción discursiva, en términos de las razones por las cuales los actores se opusieron al TLC. De acuerdo con DE, en relación con la marcha del 26 de febrero, los actores "quieren que se les tome en cuenta", así como "quieren Estado solidario", evidenciando que la protesta busca concretar una demanda relacionada con la solicitud de ser consultado y con la participación en la toma de decisiones.

En los discursos de Diario Extra (DE), ¿quiénes fueron los ausentes? Los actores no mencionados en la oposición al TLC fueron los diversos grupos religiosos que se vincularon en el proceso de lucha del referéndum, como la Conferencia Episcopal de Costa Rica (CECOR), las altas figuras de la jerarquía de la Iglesia Católica, como Monseñor Hugo Barrantes, Arzobispo de San José, u otras denominaciones religiosas. Los Comités Patrióticos tampoco protagonizaron acciones colectivas en este medio escrito. En noviembre de 2007 se mencionó al Comité Patriótico de Heredia, y la Coordinadora de Lucha contra el TLC de Heredia. En las acciones colectivas del año, no se brindó cobertura a los grupos virtuales a favor o contra del TLC.

El periódico LN mostró una mínima diversidad de actores sociales, tanto de las personas que se opusieron como de las que favorecieron la aprobación del TLC, con ciertas 
excepciones que se detallan a continuación. La mayoría de noticias que versaron sobre la aprobación y rechazo del TLC y la agenda de implementación en este medio, mostraron actores específicos, la mayoría son individuales y en pocas ocasiones se detalló a actores colectivos. Una de las formas y la más recurrente para hacer mención de los actores incidentes en las diferentes acciones colectivas reseñadas por este periódico, fue primero mencionar el nombre de la persona y luego la agrupación a qué pertenece.

\section{CONCLUSIONES}

En este trabajo se han analizado las principales características en la cobertura $y$ abordaje que La Nación (LN) y Diario Extra (DE) hicieron sobre noticias relacionadas con acciones colectivas de protesta, a favor $y$ en contra del TLC. Durante el 2007, los medios de comunicación analizados reportaron un mayor número de noticias sobre acciones colectivas relacionadas con la petición de "otras demandas", es decir, de demandas no relacionadas con el TLC. En términos generales, ambos medios reportaron más noticias en contra que a favor del TLC. Cuando se reportaron más noticias en oposición fue en los meses en que se realizaron las marchas más concurridas, en febrero, mayo $y$ setiembre. DE publicó más noticias a favor $y$ en contra del Tratado que LN.

Llama la atención que durante el periodo que incluye los meses de abril-setiembre el porcentaje de noticias con respecto al año, correspondió a un $30 \%$ a la oposición, y a un $63,64 \%$ a favor del TLC. Estos porcentajes difieren de la evolución en la intención de voto en el referéndum, incluso, como se mencionó anteriormente están a la inversa.

En las noticias donde ambos medios reportaron las mismas acciones colectivas se encontraron similitudes en cuanto a los llamados al orden, $y$ a la exaltación del pacifismo, aunque este rasgo se presentó predominantemente en LN. En la (re)construcción periodística de los actores, ambos medios utilizaron estrategias similares: particularización e individualización de los actores colectivos, exaltación de determinadas figuras políticas — vinculadas a la oposición gubernamental o a la disidencia con el PLN-, exacerbación de la participación sindical y por ende, masculinización de la protesta social. Esta fue la estrategia a la que recurrieron los medios para describir la oposición al TLC.

Lo anterior no ocurrió en el caso de la representación de las personas y grupos a favor del Tratado, ya que se presentó un movimiento sumamente diverso, y en apariencia sin una dirigencia claramente identificable. Es importante señalar que durante el primer semestre del 2007, cuando los medios necesitaron identificar la posición a favor del TLC acudieron a los representantes gubernamentales. Para el segundo semestre se identificó como representantes del grupo a favor de la aprobación a determinadas agrupaciones (Alianza Ciudadana del Sí o cámaras patronales), así como, diversos colectivos generalizados como "los campesinos", "los trabajadores textiles", "los porteadores". Además se destaca el hecho de que los grupos a favor del sí fueron representados, en particular por LN, como un grupo heterogéneo $y$ unido, frente a un movimiento disgregado, conflictivo y sin claridad en sus demandas al plantear la oposición al Tratado. En el caso de La Nación (LN), su estrategia residió en identificar al PAC, $y$ en particular a Ottón Solís, como uno de los principales organizadores de la oposición al TLC. Esto no sucedió en el caso de Diario Extra (DE), donde la participación de este partido apareció desdibujada.

Para finalizar, los grandes ausentes en estos periódicos fueron los Comités Patrióticos, de cuya participación en el proceso del referéndum se dijo sumamente poco, e incluso, cuando se le menciona no se les nombra como "comités patrióticos", sino con sinónimos aumentando su invisibilización.

\section{BIBLIOGRAFÍA}

Chacón, Lorna. "Medios de comunicación y estrategias comunicativas de los actores durante el proceso de referéndum en Costa Rica”. Ponencia presentada en el marco del Seminario de Democracia y Resistencia Social ante el debilitamiento 
del Estado-Nación. Organizado por el Posgrado Centroamericano de Ciencias Políticas. 23 de octubre de 2008.

Cuvardic, Dorde. "Construcción y representación fotográfica de los conflictos sociopolíticos: la "huelga de educadores" del 2003 en el diario La Nación". Revista de Ciencias Sociales 106-107. 2004-2005: 93-106.

Fonseca, Andrea. "Privatización y prensa escrita en Costa Rica. El caso del COMBO ICE. Un análisis de la Posición del Periódico La Nación en relación con el proyecto de Modernización del Instituto Costarricense de Electricidad durante el periodo noviembre 1999-marzo 2001". [Tesis presentada para optar por el grado de Licenciatura en Sociología]. San Pedro: Universidad de Costa Rica, 2003.

García, Anthony. "Informe final de acciones colectivas en Costa Rica 2007". Decimocuarto informe sobre el Estado de la Nación en Desarrollo Humano Sostenible. San José, Proyecto Estado de la Nación. 2008.

Martín, Kattia. "El Combo del ICE y La Nación: Análisis para un periodismo socialmente responsable". [Tesis presentada para optar por el grado de Magíster Scientae en Comunicación]. San Pedro: Universidad de Costa Rica, 2004.

Mora, Sindy. "Diez años de acciones colectivas en Costa Rica". Revista Centroamericana de Ciencias Sociales. San José: Flacso, 2008. En prensa.

Raventós, Ciska. "Mi corazón dice NO": lucha contra el TLC, confianza y legitimidad en Costa Rica. 2008. Documento inédito.

Rodríguez, Florisabel. "El Referéndum del TLC del 2007 en Costa Rica: evaluación de un instrumento ciudadano". Ponencia para el Congreso "¿Gobernanza sin desarrollo?
Repensar el bienestar en América Latina”. Agosto, 2008.

Vasilashis, Irene. "La construcción discursiva, mediática y política de la realidad social (allegro)". Discurso político y prensa escrita. España: Gedisa, 1997.

\section{FUENTES PERIODÍSTICAS CONSULTADAS}

Agüero, M. "Sindicatos convocan marcha para el 26 de febrero". Diario Extra. 09 de febrero de 2007.

Artavia, B. "Comités Patrióticos de todo el país se mantendrán en lucha”. Diario Extra. 12 de octubre de 2007.

- "El TLC es el marco de referencia para seguir creciendo". Diario Extra. 23 de abril de 2007.

Espinoza, M. "Críticas contra magistrados de sala cuarta no acaban". Diario Extra. 06 de julio de 2007.

Mora, A. L. "ANEP advierte de choque social por TLC". La Nación. 01 de marzo de 2007.

. "Conflictiva reunión del NO". La Nación. 09 de octubre de 2007.

. "Opositores al TLC boicotearían referendo si se votan leyes paralelas". $L a$ Nación. 11 de julio de 2007.

. "Agresivo paro de labores tuvo mínimo apoyo en el ICE". La Nación. 11 de noviembre de 2007.

Murillo, Á. y Agüero, M. "El No evoca a próceres para pedir 'fusilar' TLC en las urnas". $L a$ Nación. 01 de octubre de 2007.

Murillo, Á. "Grupo heterogéneo se une para el 'sî'”. La Nación. 06 de junio de 2007. 
Oviedo, E. "Marcha pacífica reunió a 23.500 opositores al TLC". La Nación. 27 de febrero de 2007.

Oviedo, E. y Mata, A. "Agresivo paro de labores tuvo mínimo apoyo en el ICE". La Nación. 27 de noviembre de 2007.

Rivera, M. "Llaman a rebelión en huelga del ICE". Diario Extra. 17 de noviembre de 2007.

"Pérdidas pueden ser de hasta $\$ 500$ millones". Diario Extra. 27 de febrero de 2007.
Ruiz, G. "A semillazos los del "No" atacan casas de diputados". Diario Extra. 09 de noviembre de 2007.

Villalobos, C. A. "Grupo anti-TLC desconoce legitimidad de Sala IV y TSE". La Nación. 06 de julio de 2007.

BASE DE DATOS

Instituto de Investigaciones Sociales, Universidad de Costa Rica. Base de datos de acciones colectivas, 2007. 
